\title{
Sucesiones y la dimensión fractal
}

\author{
Luis Manuel Hernández G. \\ Imhg@fciencias.unam.mx \\ Facultad de Ciencias \\ UNAM. México
}

\section{Resumen.}

A través de una serie de problemas, cuya solución nos sumerge en el mundo matemático de los procesos infinitos, en forma natural se llega a la idea de sucesión y límite de una sucesión. En algunos de los problemas planteados aparecen objetos geométricos, con ciertas propiedades especiales, como por ejemplo la propiedad de tener longitud infinita pero encerrar un área finita, esto nos permite hacer una "presentación" de los objetos geométricos conocidos como "fractales autosemejantes". Una de las propiedades características de un fractal es su dimensión, por lo que se introduce el concepto de dimensión fractal.

Palabras claves: Infinito, sucesión, límite, autosemejanza, fractal, dimensión fractal.

\section{Abstract.}

Through a series of problems whose solution we are immersed in the mathematical world of infinite processes, in the natural way reaches the idea of sequence and limit of a sequence. In some of the problems raised appear geometric objects, with certain special properties, such as the property of being infinite length but enclose a finite area, this allows us to make a "presentation" of the geometric objects known as "self-similar fractals". One of the characteristic properties of a fractal is its size, so it introduces the concept of fractal dimension.

KeyWords: Infinity, sequence, limit, shelfsimilarity, fractal dimension.

\subsection{Introducción}

A través de una serie de problemas, cuya solución nos sumerge en el mundo matemático de los procesos infinitos, en forma natural se llega a la idea de sucesión y límite de una sucesión. En algunos de los problemas planteados aparecen objetos geométricos, con ciertas propiedades especiales, como por ejemplo la propiedad de tener longitud infinita pero encerrar un área finita, esto nos permite hacer una "presentación" de los objetos geométricos conocidos como "fractales autosemejantes". Una de las propiedades características de un fractal es su dimensión, por lo que se introduce el concepto de dimensión fractal. 


\subsection{Problema 1. Las Torres de Hanoi.}

Este es un problema que a veces se rodea de historias impregnadas de misticismo (véase [2] y [4]), pero que en realidad es un problema planteado por el matemático francés Édouard Anatole Lucas alrededor de 1880 y se formula en los términos siguientes: Se tienen tres "postes" (figura 1.1); en uno de ellos se coloca una pila de discos y el tamaño de cada uno de ellos decrece conforme crece el número de discos.

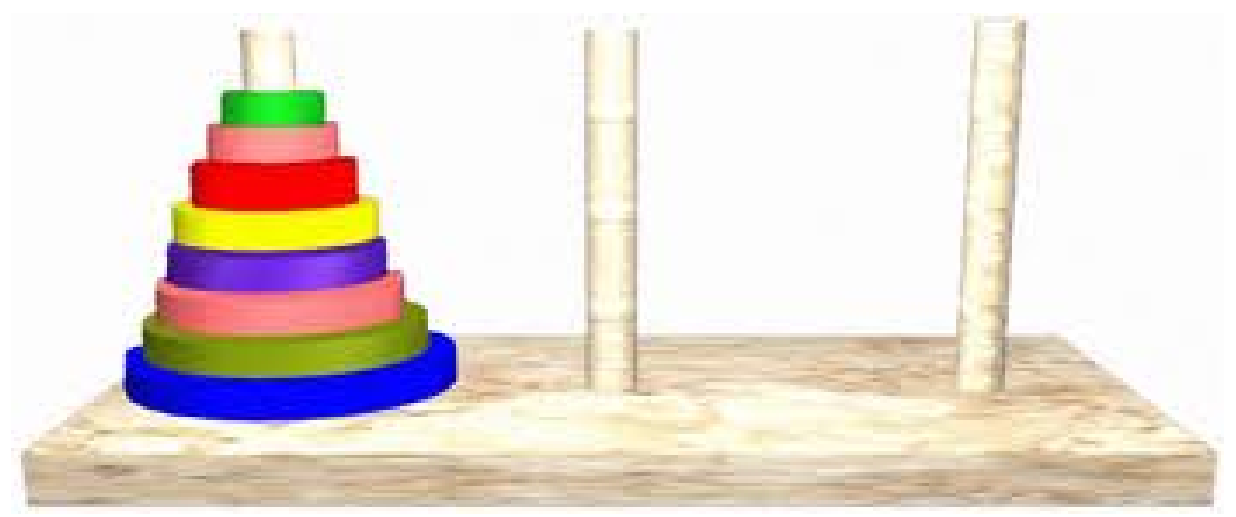

Figura 1.1 Torres de Hanoi

El proceso se debe realizar bajo las reglas siguientes: 1. En cada movimiento sólo se debe mover un disco. 2. Un disco de cierto tamaño no debe colocarse encima de uno de menor tamaño.

Denotemos por $P_{i}$ el poste inicial, $P_{a}$ el poste auxiliar, $P_{f}$ el poste final y numeremos los discos de abajo hacia arriba desde 1 hasta $n$.

Si $n=1$, el número mínimo de movimientos para cambiar la torre es igual a 1 .

Si $n=2$, el procedimiento que se sigue para cambiar de lugar la torre y obtener el número mínimo de movimientos consiste en cambiar el disco 2 al poste $P_{a}$, el disco 1 al poste $P_{f}$ y enseguida el disco 2 al poste $P_{f}$. Entonces el número mínimo de movimientos es 3 .

Si $n=3$, el procedimiento consiste en cambiar

1. El disco 3 al poste $P_{f}$

2. El disco 2 al poste $P_{a}$

3. El disco 3 al poste $P_{a}$

4. El disco 1 al poste $P_{f}$

5. El disco 3 al poste $P_{i}$

6. El disco 2 al poste $P_{f}$.

7. El disco 3 al poste $P_{f}$ 
Entonces el número mínimo de movimientos es 7.

Como se ve el procedimiento es bastante largo y tedioso, pero si usamos la información anterior y por ejemplo para el caso de 4 discos tomamos los discos 2,3 y 4 como una unidad y los movemos juntos, el problema se reduce al de $n=2$, sólo que en dos de los tres movimientos que se requieren para cambiar la torre se cambió la unidad de los tres discos juntos, lo cual significa requiere 7 movimientos cada vez. Por lo tanto el número mínimo de movimientos es 15.

Al hacer uso de este método recursivo una y otra vez, se deduce que el número mímimo de movimientos queda expresado por la fórmula

$$
N(n)=2^{n}-1
$$

donde $n$ es el número de discos. Si consideramos que $n$ pueda tomar cualquier valor natural, se tiene una sucesión $\{N(n)\}$ o $\left\{N_{n}\right\}=\left\{\frac{A_{0}}{9}, \frac{A_{0}}{9^{2}}, \frac{A_{0}}{9^{3}}, \frac{A_{0}}{9^{4}}, \cdots, \frac{A_{0}}{9^{n}}, \cdots\right\}$, cuyo término general es

$$
N_{n}=2^{n}-1
$$

\subsection{Problema 2. Triángulos anidados.}

(Véase [7]) En la figura 2 aparecen los primero triángulos de un conjunto infinito de triángulos anidados. ¿Cuánto vale la suma p de los perímetros de “todos” los triángulos?

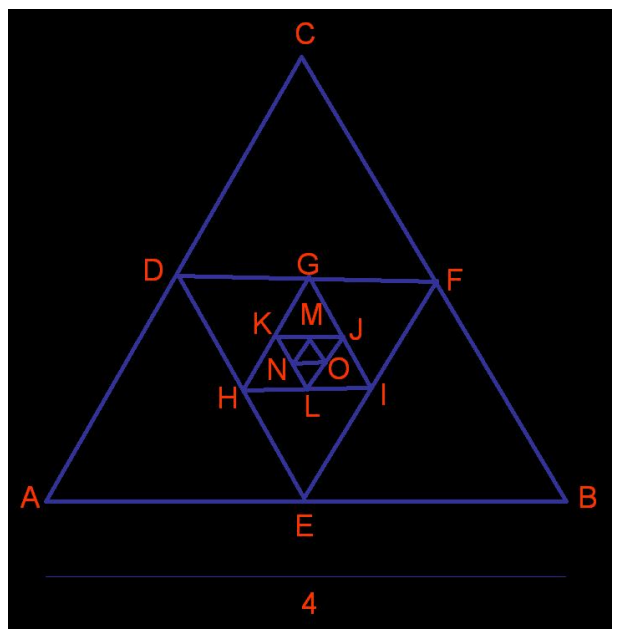

Figura 1.2 Triángulos anidados

Como cada uno de los triángulos es equilátero y el lado del más grande mide 4 , se deduce que la longitud de los triángulos que siguen es

$$
2,1, \frac{1}{2}, \frac{1}{2^{2}}, \cdots, \frac{1}{2^{n-3}}, \cdots
$$

En consecuencia se obtiene

$$
p=3\left(4+2+1+\frac{1}{2}+\frac{1}{2^{2}}+\cdots+\frac{1}{2^{n-3}}+\cdots\right)=21+\frac{3}{2}\left(\frac{1}{2}+\frac{1}{2^{2}}+\cdots+\frac{1}{2^{n-3}}+\cdots\right)
$$


es decir, $p=21+\frac{3}{2} \lim _{n \rightarrow \infty} l_{n}$, donde $l_{n}=\frac{1}{2}+\frac{1}{2^{2}}+\cdots+\frac{1}{2^{n-3}}$, es decir,

$$
1 D_{3}=D_{0}+\frac{1}{3} D_{0}+\frac{1}{3}\left(D_{0}+\frac{1}{3} D_{0}\right)+\frac{1}{3}\left(D_{0}+\frac{1}{3} D_{0}+\frac{1}{3}\left(D_{0}+\frac{1}{3} D_{0}\right)\right)=D_{0}\left(1+\frac{1}{3}\right)^{3} .
$$

La sucesión $\quad\left\{l_{n}\right\}$ tiene como término general la suma de una progresión geométrica. Como $l_{n}=$ $\frac{1-\left(\frac{1}{2}\right)^{n-2}}{1-\frac{1}{2}}=2\left(1-\left(\frac{1}{2}\right)^{n-2}\right)$, entonces $\lim _{n \rightarrow \infty} 2\left(1-\left(\frac{1}{2}\right)^{n-2}\right)=2$.

Por lo tanto $p=24$.

\subsection{Problema 3. Cuadrados anidados}

(Véase [7]) En la figura 1.3 se dibujaron los primeros cuadrados de un conjunto infinito de cuadrados anidados.

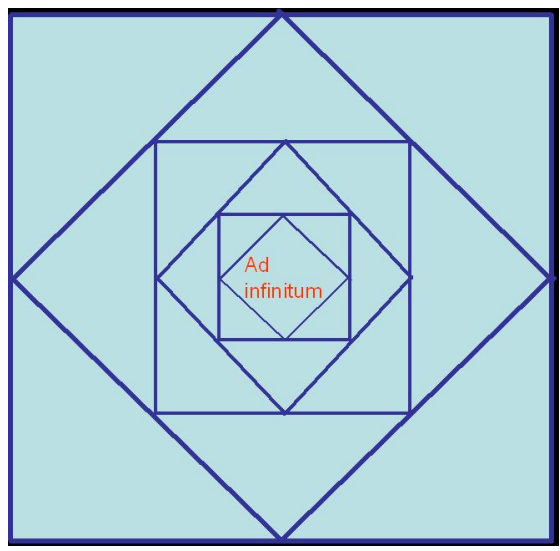

Figura 1.3 Cuadrados anidados

¿Cuál es la suma A de las áreas de "todos" los cuadrados si el área del más grande es 4?

Numeremos los cuadrados de afuera hacia adentro, $1,2,3, \cdots \frac{1}{3}, \frac{1}{3^{2}}, \frac{1}{3^{3}}, \frac{1}{3^{4}}, \cdots, \frac{1}{3^{n}}, \cdots$ Como el área del cuadrado 1 es 4, su lado mide 2, por lo que con el teorema de Pitágoras se obtiene que el lado del cuadrado 2 mide $\sqrt{2}$, entonces el área es igual a 2 , el lado del cuadrado mide $\sqrt{\left(\frac{\sqrt{2}}{2}\right)^{2}+\left(\frac{\sqrt{2}}{2}\right)^{2}}=1$. Inmediatamente nos damos cuenta que el proceso es igual para todos los cuadrados que siguen, en consecuencia la sucesión $\left\{A_{n}\right\}$ de las áreas de los cuadrados es

$$
4,2,1, \frac{1}{2}, \frac{1}{2^{2}}, \cdots, \frac{1}{2^{n-3}}, \cdots
$$

Por lo tanto la suma de "todas" las áreas es

$$
A=\lim _{n \rightarrow \infty}\left(A_{1}+A_{2}+\cdots+A_{n}\right)
$$

donde $A_{n}=\frac{1}{2^{n-3}}$, es decir, 
$A=\lim _{n \rightarrow \infty}\left(4+2+1+\frac{1}{2}+\frac{1}{2^{2}}+\cdots+\frac{1}{2^{n-3}}+\cdots\right)=6+\lim _{n \rightarrow \infty}\left(4+2+1+\frac{1}{2}+\frac{1}{2^{2}}+\cdots+\frac{1}{2^{n-3}}+\cdots\right)=8$.

\subsection{Problema 4. Sucesión de Fibonacci.}

En la obra "Liber abacci" escrita en 1202 por el matemático italiano Leonardo de Pisa, mejor conocido como Fibonacci aparecen muchos problemas matemáticos, entre los que se encuentra el siguiente: "¿Cuántas parejas de conejos se obtienen a partir de una pareja inicial en el transcurso de un año?" (Véase [8]). Se supone que:

1. 1. Una pareja de conejos se reproduce por primera vez a los dos meses y engendra una nueva pareja.

2. 2. A partir de que una pareja se reproduce por primera vez, se vuelve a reproducir mensualmente, y cada vez engendra una nueva pareja.

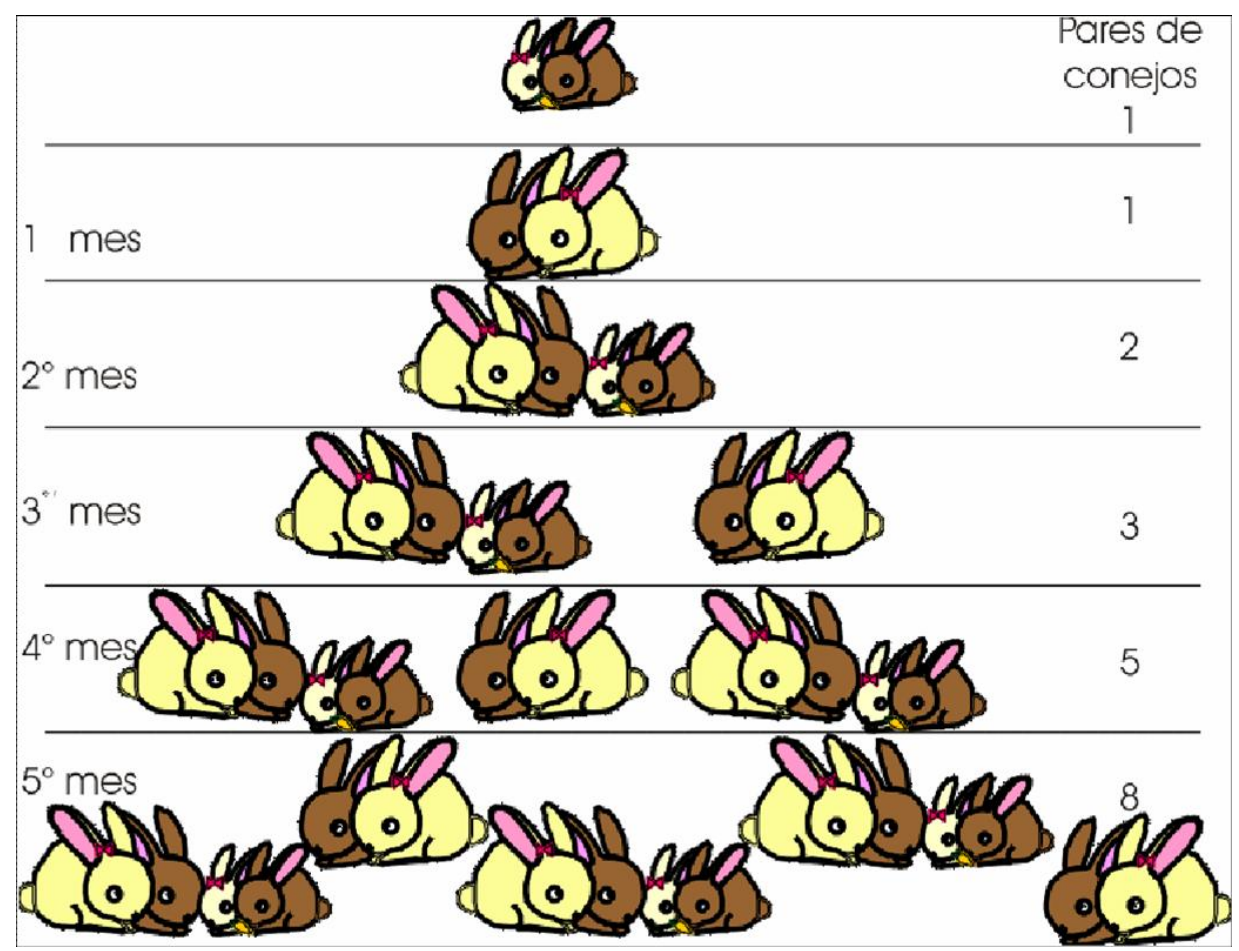

Figura 1.4

En forma inmediata se obtiene que el número de parejas en el mes $n$ está dado por una expresión del tipo $F_{n}=F_{n-2}+F_{n-1}$, para $n \geq 3$, con $F_{1}=1$ y $F_{2}=1$, luego la respuesta al problema es el valor de $F_{n}$ cuando $n=12$. Al extrapolar el problema para todo $n$, la expresión obtenida define una sucesión recurrente. 
A la sucesión $\left\{F_{n}\right\}$ se le llama sucesión de Fibonacci y a los números $F_{n}$ se les conoce como números de Fibonacci, los cuales tienen propiedades muy interesantes desde el punto de vista de la teoría de los números, escribamos algunas de ellas

1. $F_{1}+F_{2}+\cdots+F_{n}=F_{n+2}-1$

2. $F_{1}+F_{3}+F_{5} \cdots+F_{2 n-1}=F_{2 n}$

3. $F_{2}+F_{4}+F_{6} \cdots+F_{2 n}=F_{2 n+1}-1$

4. $F_{1}^{2}+F_{2}^{2}+F_{3}^{2} \cdots+F_{n}^{2}=F_{n} F_{n+1}$

5. $F_{n}^{2}=F_{n-1} F_{n+1}+(-1)^{n+1}$

6. Los números de Fibonacci consecutivos, es decir, $F_{n}$ y $F_{n+1}$, son primos entre sí.

Pero también existe una relación muy estrecha entre los números de Fibonacci y el número áureo $\phi=\frac{1+\sqrt{5}}{2}$, donde destaca el hecho sorprendente de que

$$
\lim _{n \rightarrow \infty} \frac{F_{n+1}}{F_{n}}=\phi
$$

Al número áureo también se le conoce como proporción perfecta o áurea, ya que surge de dividir un segmento que sin pérdida de generalidad se puede considerar unitario en dos partes, de tal forma que la unidad sea a la más grande, llamémosla $x$, como $x$ a $1-x$, es decir,

$$
\frac{1}{x}=\frac{x}{1-x}
$$

lo cual nos lleva a la ecuación $x^{2}+x-1=0$, cuya solución es $x=\frac{\sqrt{5}-1}{2}$, de donde se obtiene

$$
\phi=\frac{1}{x}=\frac{1+\sqrt{5}}{2}
$$

proporción que desde la antigua Grecia con el arquitecto Fidias, quien la usó para diseñar el Partenón, se siguió usando por pintores, arquitectos, escultores y músicos, en la elaboración de algunas de sus obras, y por los biólogos en el estudio de la Naturaleza, en particular en taxonomía vegetal. 

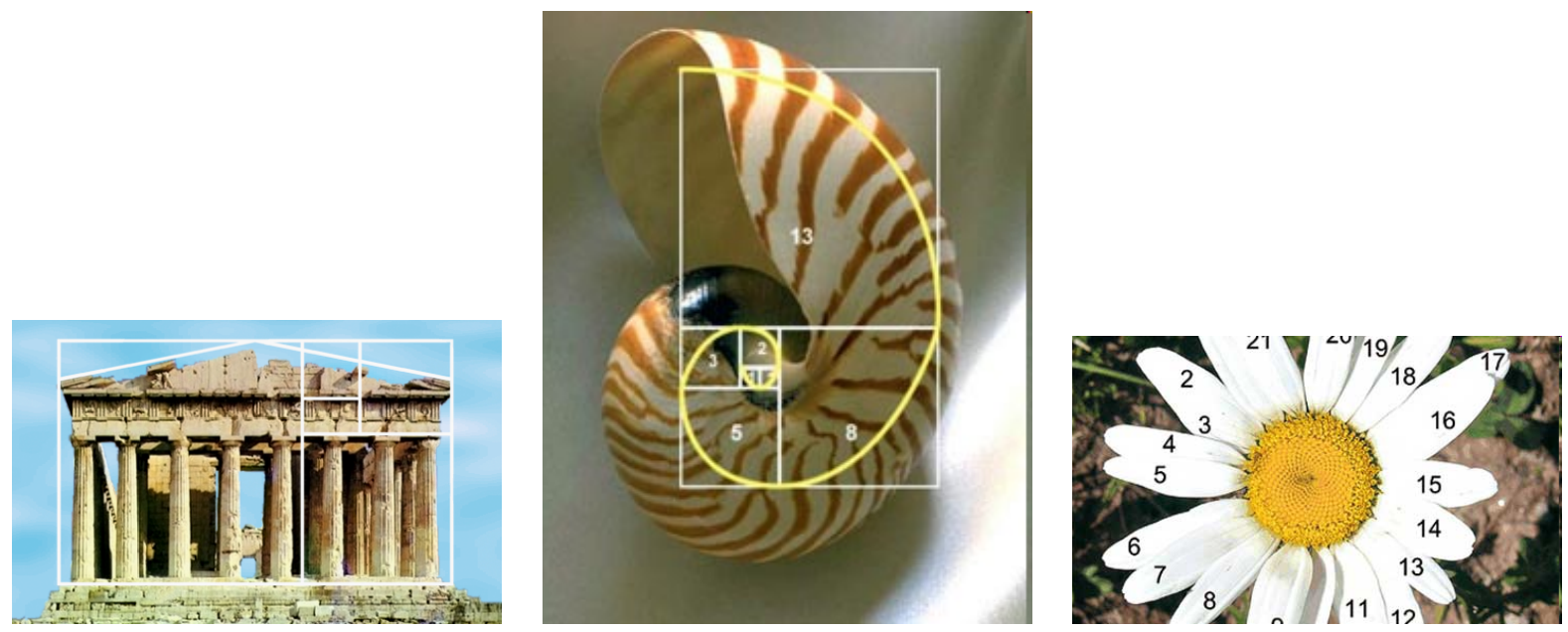

Figura 1.5

\subsection{Problema 5. Inversiones}

(Véase [8]). Consideremos una cantidad de dinero $D_{0}$ que arroja un interés anual de $100 \%$. ¿Cuál es la cantidad de dinero D al final de un año, si se considera que la reinversión es instantánea?

Si se considera que el dinero se reinvierte sólo una vez al año, entonces se obtiene $D_{1}=D_{0}+D_{0}=2 D_{0}$. Al invertirlo dos veces al año, se obtiene $D_{2}=D_{0}+\frac{1}{2} D_{0}+\frac{1}{2}\left(D_{0}+\frac{1}{2} D_{0}\right)=D_{0}\left(1+\frac{1}{2}\right)^{2}$. Si se invierte tres veces en el año, se obtiene

$$
D_{3}=D_{0}+\frac{1}{3} D_{0}+\frac{1}{3}\left(D_{0}+\frac{1}{3} D_{0}\right)+\frac{1}{3}\left(D_{0}+\frac{1}{3} D_{0}+\frac{1}{3}\left(D_{0}+\frac{1}{3} D_{0}\right)\right)=D_{0}\left(1+\frac{1}{3}\right)^{3}
$$

Entonces al invertirlo $n$ veces en el año, se obtiene $D_{n}=D_{0}\left(1+\frac{1}{n}\right)^{n}$

Por lo tanto si la reinversión es instantánea se tiene que $D=D_{0} \lim _{n \rightarrow \infty}\left(1+\frac{1}{n}\right)^{n}$.

De nuevo aquí aparece también una sucesión, en este caso $\left\{D_{n}\right\}$, donde el término general de la sucesión es $D_{n}=\left(1+\frac{1}{n}\right)^{n}$.

Como

$$
\lim _{n \rightarrow \infty} D_{n}=\lim _{n \rightarrow \infty}\left(1+\frac{1}{n}\right)^{n}=e \approx 2.7182818284590452354
$$

(este número, que se conoce como número $\boldsymbol{e}$, es uno de los números más importantes no sólo en Matemáticas sino en la Ciencia en general, el cual es un número irracional y trascendente), entonces $D=D_{0} e$.

\subsection{Problema 6. curva de Koch.}


(Véase [6]) En las figuras 1.6-1.7 aparecen los primeros pasos de de un proceso infinito de construcción de una curva que se conoce como curva de Koch en honor del matemático Niels Fabián Helge von Koch, y en la figura 5 las primeras etapas de la construcción de una curva llamada curva copo de nieve, por su forma característica o también conocida como copo de nieve de Koch.

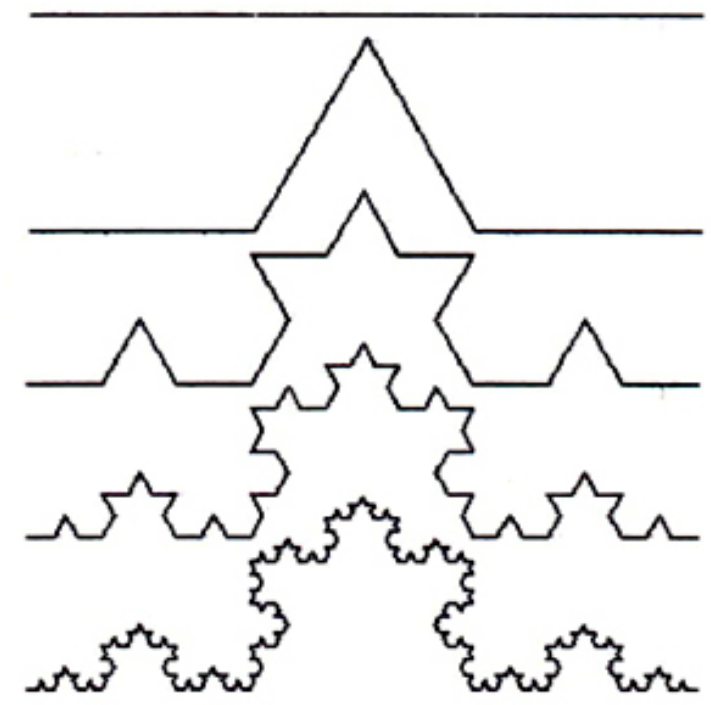

Figura 1.6 Curva de Koch

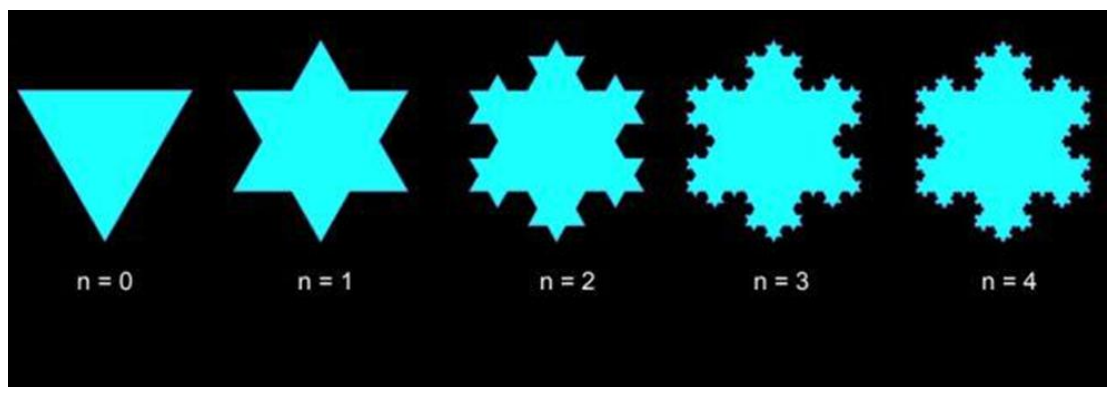

Figura 1.7 Curva copo de nieve

¿Cuál es la longitud de la curva de Koch si la longitud del segmento inicial es $\ell ?$ ¿Cuál es el área encerrada por la curva copo de nieve si el área del triángulo original es $A_{0}$ ?

En cada etapa de construcción de la curva de Koch, el número de segmentos que aparecen es igual a

$$
1,4,4^{2}, 4^{3}, \cdots, 4^{n-1}, \cdots
$$

cada uno de ellos de longitud

$$
l, \frac{\ell}{3}, \frac{\ell}{3^{2}}, \frac{\ell}{3^{3}}, \frac{\ell}{3^{4}}, \cdots, \frac{\ell}{3^{n}}, \cdots
$$

respectivamente. Por lo tanto su longitud es 


$$
L=\ell \lim _{n \rightarrow \infty}\left(\frac{4}{3}\right)^{n-1}="+\infty "
$$

Para la curva copo de nieve, en cada etapa de su construcción, al triángulo inicial se le agregan sucesivamente

$$
3,3 \times 4,3 \times 4^{2}, 3 \times 4^{3}, \cdots, 3 \times 4^{n-1}, \cdots
$$

triángulos, cada uno de ellos de área

$$
\frac{A_{0}}{9}, \frac{A_{0}}{9^{2}}, \frac{A_{0}}{9^{3}}, \frac{A_{0}}{9^{4}}, \cdots, \frac{A_{0}}{9^{n}}, \cdots
$$

respectivamente. Por lo tanto el área encerrada por la curva copo de nieve es

$$
A=A_{0}\left(1+\frac{3}{9} \lim _{n \rightarrow \infty}\left(1+\frac{4}{9}+\left(\frac{4}{9}\right)^{2} \cdots+\left(\frac{4}{9}\right)^{n-1}\right)\right)=\frac{8}{5} A_{0}
$$

\subsection{Problema 7. Triángulo de Sierpinski.}

(Véase [5]) En la figura 1.8 están los primeros pasos del proceso infinito de construcción del llamado triángulo de Sierpinski (Waclaw Sierpinski, matemático polaco (1882-1969)), cuya construcción se "hace" quitando sucesivamente el triángulo central de los triángulos negros, a partir de un triángulo dado.

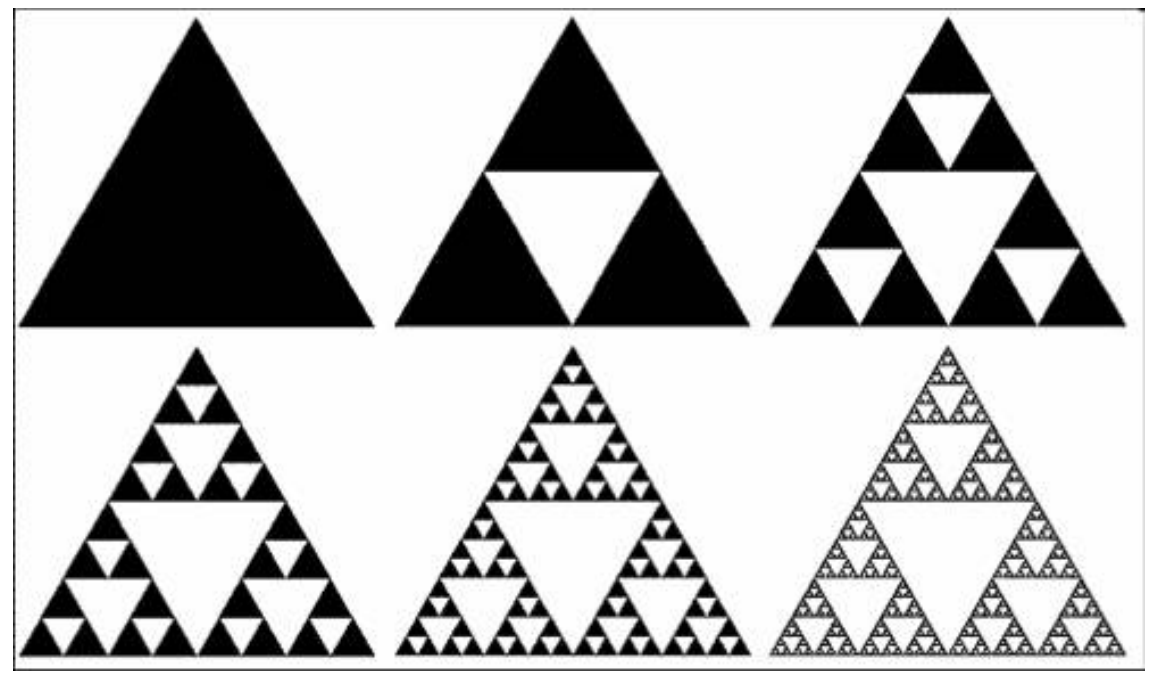

Figura 1.8

¿Cuál es el área encerrada por el triángulo de Sierpinski si el triángulo inicial tiene área 1 ?

Al triángulo original se le van quitando sucesivamente

$$
1,3,3^{2}, 3^{3}, \cdots, 3^{n-1}, \cdots
$$

triángulos, cada uno de ellos con un área igual a 


$$
\frac{1}{4}, \frac{1}{4^{2}}, \frac{1}{4^{3}}, \frac{1}{4^{4}}, \cdots, \frac{1}{4^{n}}, \cdots
$$

respectivamente. Por lo tanto

$$
A=1-\frac{1}{4} \lim _{n \rightarrow \infty}\left(1+\frac{3}{4}+\left(\frac{3}{4}\right)^{2}+\cdots+\left(\frac{3}{4}\right)^{n-1}\right)=0 .
$$

\subsection{Problema 8. Tapete de Sierpinski}

(Véase [5]) En la figura 1.9 están los primeros pasos de un proceso infinito de construcción del llamado tapete de Sierpinski, cuya construcción se "hace" quitando sucesivamente el cuadrado central de los cuadrados negros, a partir de un cuadrado inicial.
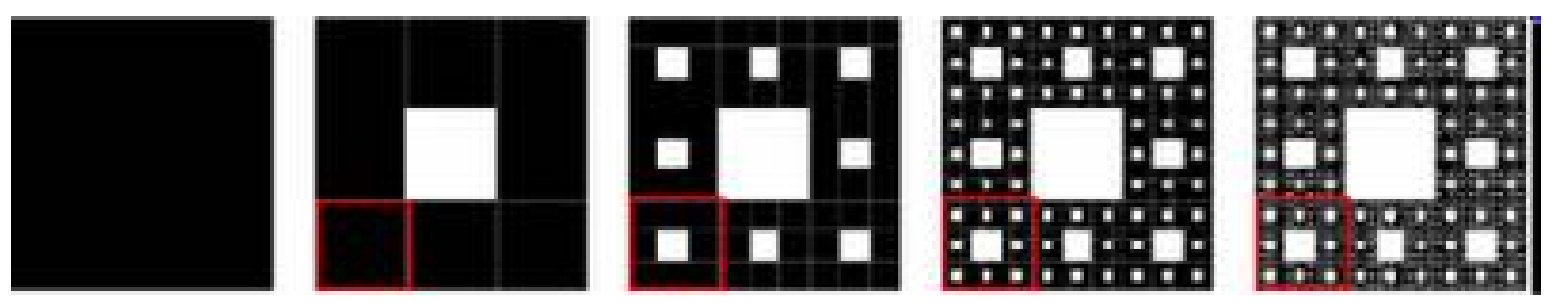

Figura 1.9 Tapete de Sierpinski.

¿Cuál es el área del tapete de Sierpinski, si el área del cuadrado inicial es 1 ?

En este caso al cuadrado inicial se le van quitando sucesivamente

$$
1,8,8^{2}, 8^{3}, \cdots, 8^{n-1}, \cdots
$$

cuadrados, cada uno de ellos con un área igual a

$$
\frac{1}{9}, \frac{1}{9^{2}}, \frac{1}{9^{3}}, \frac{1}{9^{4}}, \cdots, \frac{1}{9^{n}}, \cdots
$$

respectivamente. Por lo tanto

$$
A=1-\frac{1}{9} \lim _{n \rightarrow \infty}\left(1+\frac{8}{9}+\left(\frac{8}{9}\right)^{2}+\cdots+\left(\frac{8}{9}\right)^{n-1}\right)=0
$$

\subsection{Problema 9. Esponja de Menger}

. (Véase [6]) En la figura 1.10 se ilustran los primeros pasos de un proceso infinito de construcción de la llamada esponja de Menger (Karl Menger 1840-1921, matemático austriaco), cuya construcción se "hace" quitando sucesivamente el bloque central de cubos respecto de cada cara, a partir de un cubo inicial. 


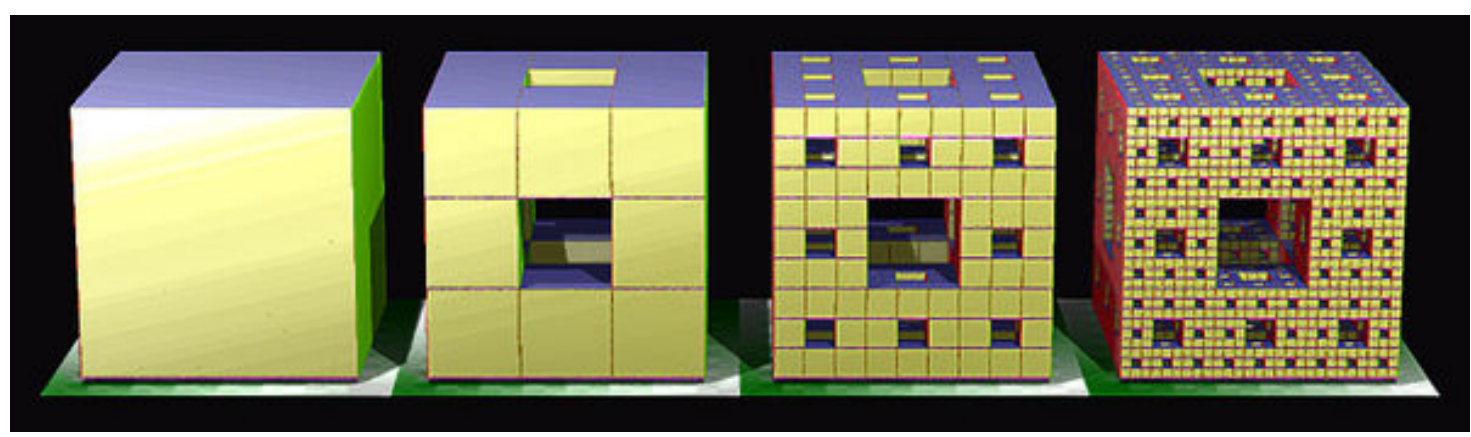

Figura 1.10 Esponja de Menger.

¿Cuál es el volumen de la esponja de Menger si el volumen del cubo inicial es 1 ?

Aquí, al cubo inicial se le van quitando sucesivamente

$$
7,7 \times(20), 7 \times(20)^{2}, 7 \times(20)^{3}, \cdots, 7 \times(20)^{n-1}, \cdots
$$

cubos, cada uno de ellos con un volumen igual a

$$
\frac{1}{27}, \frac{1}{(27)^{2}}, \frac{1}{(27)^{3}}, \frac{1}{(27)^{4}}, \cdots, \frac{1}{(27)^{n}}, \cdots
$$

respectivamente. Por lo tanto

$$
V=1-\frac{7}{27} \lim _{n \rightarrow \infty}\left(1+\frac{20}{27}+\left(\frac{20}{27}\right)^{2}+\cdots+\left(\frac{20}{27}\right)^{n-1}\right)=0
$$

\subsection{Problema 10. Conjunto de Cantor}

Problema 10. (Véase [3]) En la figura 1.11 aparecen los primeros pasos del proceso infinito de construcción de un conjunto que se conoce como conjunto de Cantor, en honor del matemático alemán George Cantor (1845-1918), cuya construcción se "hace" quitando el segmento central sin sus extremos de los tres que resultan al dividir un segmento dado en tres partes iguales y después sucesivamente se aplica el mismo procedimiento con los segmentos que van sobreviviendo. 


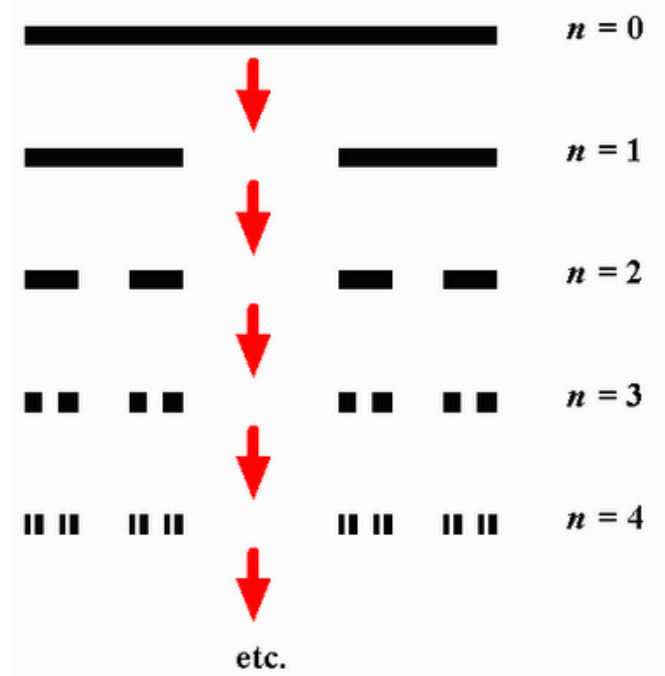

Figura 1.11 Conjunto de Cantor

¿Cuál es la longitud del conjunto de Cantor si la longitud del segmento original es 1 ?

En cada etapa de la construcción del conjunto de Cantor, al segmento inicial sucesivamente se le quitan

$$
1,2,2^{2}, 2^{3}, \cdots, 2^{n-1}, \cdots
$$

segmentos, cada uno de ellos de longitud igual a

$$
\frac{1}{3}, \frac{1}{3^{2}}, \frac{1}{3^{3}}, \frac{1}{3^{4}}, \cdots, \frac{1}{3^{n}}, \cdots
$$

respectivamente.

Por lo tanto la longitud del conjunto de Cantor es

$$
l=1-\frac{1}{3} \lim _{n \rightarrow \infty}\left(1+\frac{2}{3}+\left(\frac{2}{3}\right)^{2}+\cdots+\left(\frac{2}{3}\right)^{n-1}\right)=0 .
$$

En los cinco problemas finales aparecen objetos que a todas las escalas posibles se ven idénticos al objeto más grande, es decir, a diferentes escalas y en cualquier punto del objeto aparecen una y otra vez copias del objeto. Este tipo de entes geométricos, que entre otras, tienen la propiedad de que su dimensión no necesariamente es un número entero, se conocen como fractales autosemejantes.

En los últimos 5 problemas ya no se hizo un énfasis explícito en las sucesiones que aparecen al resolverlos para no ser redundantes, pero como se ve, algunas de ellas son parecidas a las que aparecen en los problemas anteriores

\subsection{Dimensión fractal.}

En la figura 1.12 aparecen los elementos característicos (un segmento, un área y un volumen) de los espacios topológicos de dimensión 1,2 y 3 respectivamente. 


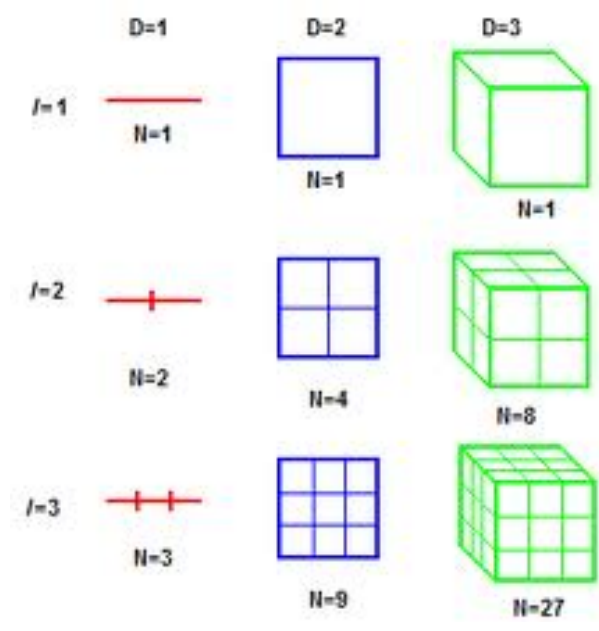

Figura 1.12 Dimensión topológica.

Si los segmentos en cada una de las dimensiones $D=1, \mathrm{D}=2$ y $\mathrm{D}=3$ se dividen en un número $i$ de partes iguales, el número $N$ de figuras iguales (segmentos, cuadrados o cubos) que se obtiene en cada caso es

$$
N=i^{D}
$$

De la expresión anterior se deduce en forma inmediata que

$$
D=\frac{\ln N}{\ln i}
$$

Motivado por este hecho se generaliza el concepto de dimensión de autosemejanza o dimensión fractal para objetos como los que se obtienen en los últimos cinco problemas, a través de la fórmula

$$
D=\frac{\ln N}{\ln i}
$$

Para cada uno de los fractales autosemejantes obtenidos en los cinco problemas últimos, después de identificar en cada caso cual es el valor de $i$ y de $N$ se obtiene:

1. Para la curva de Koch se tiene que $i=3$ y $N=4$, por lo que su dimensión es $D=\frac{\ln 4}{\ln 3} \simeq 1.2619$

2. En el triángulo de Sierpinski, los valores correspondientes son $i=2$ y $N=3$, en consecuencia su dimensión es

$$
D=\frac{\ln 3}{\ln 2} \simeq 1.5850
$$

3. En el tapete de Sierpinski los valores son $i=3$ y $N=8$, por lo tanto su dimensión es

$$
D=\frac{\ln 8}{\ln 3} \simeq 1.8928
$$

4. Para la esponja de Menger se tiene que $i=3$ y $N=20$, por lo tanto el valor que se obtiene para su dimensión es

$$
D=\frac{\ln 20}{\ln 3} \simeq 2.7268
$$

5. Mientras que para el conjunto de Cantor, como $i=3$ y $N=2$, en consecuencia su dimensión es

$$
D=\frac{\ln 2}{\ln 3} \simeq 0.6309
$$




\section{Bibliografía}

[1] Boyce William E., DiPrima Richard C. (1983). Ecuaciones Diferenciales y problemas con valores en la frontera. Editorial Limusa, México.

[2] Gamow George. (1969). Uno dos tres ... infinito, Espasa Calpe, Madrid.

[3] Morris W. Hirsch, Stephen Smale, Robert L. Devaney. (2004). Differential equations, dynamical systems, and an introduction to chaos. San Diego California, Academic

[4] Kasner Edward, Newman James. (2001). Mathematic and Imagination. Mineola, New York, Dover.

[5] Peitgen Heinz-Otto., Jurgens Hartmut, Saupe Dietmar. (2000). Chaos and Fractals: New Frontiers of Science. Springer-Verlag, New York.

[6] Stewart Ian. (1995) Does God Play Dice?: The Mathematics of Chaos. Penguin books.

[7] Vilenkin Alexander. (1985). Matemáticas II. (en ruso), Vyshya Shkola, Moscú.

[8] Vorobiov N. N. (1978). Lecciones Populares de Matemáticas. Números de Fibonacci. Editorial Mir, Moscú. 